\title{
Renal cell carcinoma of native kidney in Chinese renal transplant recipients: a report of 12 cases and a review of the literature
}

\author{
Chi Yuen Cheung $\cdot$ Man Fai Lam • King Chung Lee • \\ Gavin Sheung Wai Chan - Kwok Wah Chan - Ka Foon Chau \\ Chun Sang Li • Tak Mao Chan · Kar Neng Lai
}

Received: 29 November 2010/ Accepted: 5 February 2011/Published online: 10 March 2011

(C) The Author(s) 2011. This article is published with open access at Springerlink.com

\begin{abstract}
Objectives To present and discuss the epidemiological and clinical aspects, as well as therapeutic options and outcome of de novo renal cell carcinoma (RCC) of the native kidneys in a series of Chinese renal transplant recipients.

Patients and Methods A retrospective, cohort study examining all renal transplant recipients with the diagnosis of RCC of native kidney followed up in two major regional hospitals in Hong Kong between January 2000 and December 2009. Clinical data included age, gender, cause of renal failure, symptoms at presentation, duration of transplantation, immunosuppressive therapy, and history of acquired cystic
\end{abstract}

C. Y. Cheung - K. F. Chau - C. S. Li

Renal Unit, Department of Medicine,

Queen Elizabeth Hospital, Kowloon, Hong Kong

M. F. Lam · T. M. Chan · K. N. Lai ( $\square)$

Renal Unit, Department of Medicine,

Queen Mary Hospital, The University of Hong Kong,

102 Pokfulam Road, Pok Fu Lam, Hong Kong

e-mail: knlai@hkucc.hku.hk

K. C. Lee

Department of Pathology, Queen Elizabeth Hospital,

Kowloon, Hong Kong

G. S. W. Chan · K. W. Chan

Department of Pathology, Queen Mary Hospital,

Pok Fu Lam, Hong Kong kidney disease (ACKD). Laboratory, radiographic, operative, and pathology reports were used to assess the tumor extent.

Results Among the 1,003 renal transplant recipients recruited, 12 transplant recipients had a nephrectomy for a total of $13 \mathrm{RCC}$. The prevalence of de novo RCC was $1.3 \%$. The mean age at diagnosis of RCC was 48.4 years, and the median time from transplantation to diagnosis was 6.1 years. ACKD was found in $6(50 \%)$ of the patients. All patients except one were asymptomatic. pT1 disease was found in ten patients with a mean tumor size of $3.2 \mathrm{~cm}$. All patients were treated successfully with radical nephrectomy. After a median follow-up of 38 months, two patients $(16.7 \%)$ died. One died of sepsis, and the other died of metastatic carcinoma.

Conclusions With increasing data showing a better prognosis if RCC is detected early by screening, it is time to consider screening all kidney transplant recipients for ACKD and RCC.

Keywords Acquired cystic kidney disease -

Chinese $\cdot$ Kidney transplantation .

Renal cell carcinoma - Ultrasound

\section{Introduction}

Renal transplantation remains the best treatment option for patients with end stage renal disease (ESRD) for their survival and quality of life. There is 
a significant increased risk of malignancy, most common skin malignancies and non-Hodgkin lymphomas, in renal transplant recipients as a result of the use of immunosuppressive medication [1]. On the other hand, carcinoma of the native kidney accounts for less than $5 \%$ of all malignancies found in transplant recipients [2]. After successful renal transplantation, the risk of developing renal cell carcinoma (RCC) in native kidneys is about 15 times higher than in the normal population [1].

There has been only a few reported series comprising few renal transplant patients with RCC of native kidneys due to the relative rarity of the condition. Furthermore, there is scarcity of such data in the Asian population. In this study, we present and discuss the epidemiological and clinical aspects, as well as therapeutic options and outcome of de novo RCC of the native kidneys in our renal transplant recipients.

\section{Patients and methods}

This is a retrospective, cohort study examining all renal transplant recipients with the diagnosis of RCC of native kidney followed up in two major regional hospitals in Hong Kong, Queen Mary Hospital and Queen Elizabeth Hospital, between January 2000 and December 2009. During the time period examined, there was no protocol of including routine ultrasound monitoring of native kidney in all patients as a part of follow-up. Clinical, pathological, and follow-up data were retrieved from each patient's medical record. Clinical data included age, gender, cause of renal failure, symptoms at presentation, duration of dialysis, duration of transplantation, immunosuppressive therapy, and history of acquired cystic kidney disease (ACKD). ACKD was defined as three or more cysts per kidney on ultrasound scan [3]. Laboratory, radiographic, operative, and pathology reports were used to assess the tumor extent. Tumor stage was determined according to the 2002 TNM classification [4]. The subtype of the tumor was classified according to the Heidelberg classification of the kidney tumors [5]. Tumors were graded according to the Fuhrman scheme [6]. Continuous data are expressed as means \pm standard deviation (SD) while categorical data are expressed as percentages.

\section{Results}

Among the 1,003 renal transplant recipients recruited, 12 transplant recipients had a nephrectomy for a total of 13 RCC. The prevalence of de novo RCC in our present series was $1.3 \%$. The demographic and clinicopathologic characteristics of our patients were shown in Table 1 and 2. There were 10 men and two women with mean age of 40 years (SD 10.6 years; range 27.5-68.3 years) at the time of transplant. Nine patients

Table 1 Demographic data of renal transplant recipients with RCC

\begin{tabular}{|c|c|c|c|c|c|c|}
\hline $\begin{array}{l}\text { Patient } \\
\text { number }\end{array}$ & Gender & ESRD & $\begin{array}{l}\text { Age at diagnosis } \\
\text { of RCC (years) }\end{array}$ & $\begin{array}{l}\text { Interval between } \\
\text { transplant and RCC } \\
\text { diagnosis (months) }\end{array}$ & ACKD & Immunosuppressive regimen \\
\hline 1 & M & Chronic GN & 46 & 66 & - & Pred + Tac + MMF \\
\hline 2 & $\mathrm{~F}$ & Unknown & 70 & 15 & + & Pred + Tac + Aza \\
\hline 3 & M & Chronic GN & 47 & 46 & - & Pred + CsA \\
\hline 4 & M & Unknown & 48 & 147 & - & Pred + CsA \\
\hline 5 & M & Unknown & 40 & 72 & - & Pred + Tac + MMF \\
\hline 6 & M & Chronic GN & 55 & 128 & - & Pred + CsA \\
\hline 7 & M & Chronic GN & 48 & 189 & - & Pred + CsA \\
\hline 8 & M & Chronic GN & 52 & 56 & + & Pred + Tac + MMF \\
\hline 9 & M & Unknown & 41 & 104 & + & Pred + CsA \\
\hline 10 & $\mathrm{~F}$ & Unknown & 43 & 40 & + & Pred + CsA + MMF \\
\hline 11 & M & Unknown & 42 & 75 & + & Pred + CsA \\
\hline 12 & M & Unknown & 49 & 258 & + & Pred + CsA \\
\hline
\end{tabular}

ESRD end stage renal disease, $R C C$ renal cell carcinoma, GN glomerulonephritis, ACKD acquired cystic kidney disease, Pred prednisolone, $T a c$ tacrolimus, $M M F$ mycophenolate mofetil, $C s A$ cyclosporine 
Table 2 Clinicopathologic characteristics of renal transplant recipients with RCC

\begin{tabular}{lllllll}
\hline $\begin{array}{l}\text { Patient } \\
\text { number }\end{array}$ & $\begin{array}{l}\text { Tumor } \\
\text { location }\end{array}$ & $\begin{array}{l}\text { Tumor } \\
\text { size }(\mathrm{cm})\end{array}$ & Type & Stage, Fuhrman & Follow-up (month) & Treatment \\
\hline 1 & RK & 2 & Clear cell & T1N0M0, G2 & 85 (Died) & Lap nephrectomy \\
2 & LK & 5 & Clear cell & T1N0M0, G1 & 32 (Alive) & Open nephrectomy \\
3 & LK & 2.3 & Clear cell & T1aN0M0, G2 & 25 (Alive) & Open nephrectomy \\
4 & RK & 6 & Papillary & T1N0M0, G2 & 119 (Alive) & Open nephrectomy \\
& LK & 1.5 & Papillary & T1N0M0, G2 & & Open nephrectomy \\
5 & LK & 4 & Papillary & T1N0M0, G1 & 68 (Alive) & Open nephrectomy \\
6 & RK & 1.7 & Clear cell & T1N0M0, G2 & 45 (Alive) & Open nephrectomy \\
7 & LK & 3 & Clear cell & T1N0M0, G2 & 21 (Alive) & Open nephrectomy \\
8 & RK & 5.9 & Papillary & T1N0M0, G2 & 18 (Alive) & Open nephrectomy \\
9 & LK & 4 & Papillary & T1N0M0, G1 & 73 (Alive) & Open nephrectomy \\
10 & LK & 2.5 & Papillary & T2N1M0, G3 & 12 (Died) & Open nephrectomy \\
11 & RK & 1 & Clear cell & T1N0M0, G2 & 50 (Alive) & Open nephrectomy \\
12 & RK & 3.2 & Clear cell & T2N0M0, G3 & 4 (Alive) & Lap nephrectomy \\
\hline
\end{tabular}

$L K$ left kidney, $R K$ right kidney, Lap laparoscopic

received deceased kidney transplantation, and the remaining three received kidneys from living donors. The mean age at diagnosis of RCC was 48.4 years (SD 8 years; range 39.6-69.5 years), and the median time from transplantation to diagnosis of malignancy was 6.1 years (range 1.2-21.5 years). The underlying causes of end-stage renal failure were chronic glomerulonephritis in five patients and unknown in the remaining seven. ACKD was found in $6(50 \%)$ of the patients.

Only one patient presented with gross hematuria while the remaining patients were totally asymptomatic. None of them had polycythemia. All RCC were detected by ultrasound and later confirmed by computed tomography. The ultrasound was arranged when a post-transplant medical or surgical complication was suspected during follow-up.

For maintenance immunosuppressive therapy before the diagnosis of RCC, five received triple immunosuppressive therapy (all were on corticosteroids, one on cyclosporine, four on tacrolimus, one on azathioprine, and four on mycophenolate mofetil) and seven patients received two immunosuppressive agents (all were on corticosteroids and cyclosporine). None of them had induction therapy. Four patients received treatment for acute cellular rejection before the diagnosis of RCC.

Five tumors were in the right kidney and six in the left; while the remaining patient had bilateral tumors.
The histology included seven clear cell RCC (54\%) and six papillary RCC (46\%). All the papillary RCC were type 1 . The mean (SD, range) tumor size was $3.2(1.6,1-6) \mathrm{cm}$. There were three, eight, and two Furhman nuclear Grade 1, 2, and 3 tumors, respectively. Eleven lesions were classified as pT1N0M0, one as pT2N0M0, and one as pT2N1M0. Only one tumor was metastasized at diagnosis. All patients were treated successfully with radical nephrectomy. Two had laparoscopic radical nephrectomy. The patient who had bilateral RCC was treated with bilateral nephrectomy.

Immunosuppressive therapy was modified in all patients after the diagnosis of RCC with dosage reduction in calcineurin inhibitors. For the four patients receiving mycophenolate mofetil, the medication was withdrawn in three and reduced in the remaining patient. Azathioprine was withdrawn in the single patient on azathioprine. On the other hand, sirolimus was introduced for immunosuppression in ten patients after nephrectomy.

After a median (range) follow-up of 38 (4-118) months, two patients (16.7\%) died. One died of sepsis, and the other died of metastatic carcinoma 11 months after the diagnosis of RCC. The remaining patients were alive with no evidence of recurrence of malignancy at the time of the current report. 


\section{Discussion}

The prevalence of native kidney RCC after renal transplantation in our locality is $1.3 \%$ - a figure similar to that reported in literature which varies from 0.34 to $5 \%$, depending on whether ultrasound screening was performed $[3,7,8]$. ACKD has been described in native kidneys of patients on long-term dialysis, with RCC being diagnosed with increasing frequency in patients with chronic renal failure. In most, but not all, instances the cancers develop in association with ACKD. It has been suggested that ACKD is an independent risk factor for RCC $[9,10]$. In patients with ACKD, the prevalence is $19 \%$ and in patients with complex cysts, it is $54 \%$ [8]. Papillary hyperplasia of cyst epithelium is recorded in virtually every detailed pathology report of tumors arising in ACKD and is the likely pathogenetic basis for the development of renal tumors in cystic kidneys complicating dialysis [11]. However, the prevalence of ACKD and the behavior of cyst development after renal transplantation are not well understood. In the current series, half of our patients with RCC were found to have ACKD. Since we have not performed ultrasound examination for all our renal transplant recipients, it is difficult to conclude that ACKD is associated with a higher prevalence of native kidney RCC. Nevertheless, patients without ACKD may also develop RCC after kidney transplant. A recent study showed that the occurrence of native renal cysts that is even a single cyst confers a 1.7-fold higher risk of developing RCC in renal transplant recipients [12]. Similar to other studies [13, 14], RCC was diagnosed at a younger age in renal transplant recipients (mean age: 48.4 years in our series) than in the general population (62.6 years) [15]. This is probably related to the higher prevalence of ACKD and higher frequency of ultrasound examination in renal transplant population.

The prevalence of ACKD in renal transplant recipients is lower than that in dialysis patients [8], possibly related to the better renal function in renal transplant recipients resulting in a slower cystic development. The influence of immunosuppressive agents on cyst formation remains poorly understood. Unlike other locations, immunosuppression appears not to increase the risk of developing malignant changes in native kidneys, as suggested by the identical incidence of RCC in heart transplant patients and the general population. One may argue that the development of post-renal transplant RCC is more likely related to ESRD and dialysis therapy [16].
The diagnosis of native kidney RCC in renal transplant recipients is difficult. It is typically an incidental finding during ultrasound scan or computed tomography for other clinical indications. In fact, most tumors are small and asymptomatic at presentation. In a retrospective study, the median native kidney tumor size was $2 \mathrm{~cm}$ (pathologic T classification: 12 pT1, 3 pT2, and 7 pT3) [17]. In another study in which systematic ultrasound of native kidneys was performed for 129 renal transplant recipients, 5 tumors, with a mean size of $2.5 \mathrm{~cm}$, were diagnosed. All of the tumors were pT1 lesions [3]. In our current series, most patients (11 of 12) were asymptomatic except one presenting with gross hematuria. Similarly, pT1 disease was found in 10 patients with a mean tumor size of $3.2 \mathrm{~cm}$. The observation of a higher prevalence of early-stage tumors in our transplant recipients compared with the general population is in accord with other studies $[3,17]$.

The percentage of clear cell RCC (54\%) and papillary RCC $(46 \%)$ in our series is similar to that reported in literature. The incidence of renal papillary tumors is notably higher in hemodialysis and transplant patients than in the general population in which clear cell RCC accounts for 75-85\% of tumors [18]. In contrast, papillary RCC represents 36 and $40 \%$ of the histopathology in two Japanese series of renal transplant recipients [17, 18]. The difference in histological distribution between RCC in transplant recipients and general population is probably related to the presence of ACKD in dialysis and transplant populations. Moreover, it may be related to the genetic mechanism. While sporadic papillary RCC in the general population usually shows allelic duplications of chromosomes 7 and 17 [19], this is not common in RCC related to ESRD. Moreover, RCC related to ESRD is typically not associated with p53 gene mutation [10].

Surgery is the preferred treatment as it is curative for the majority of RCC without metastasis [20]. Radical nephrectomy was performed for all of our patients and the patient with bilateral RCC underwent bilateral nephrectomy. Besides surgery, minimizing immunosuppression with the administration of mammalian target of rapamycin inhibitors (m-TORi) represents another approach to the management of de novo RCC. m-TORi provide a strong cancer preventive effect by blocking the growth and proliferation of tumor cells, downregulating the PI3 K-Akt-mTOR pathway 
(frequently activated in cancer), and finally suppressing angiogenesis [21]. Among tumors using the mTOR pathway for activation, RCC is probably the most sensitive tumor to m-TORi [22]. Both temsirolimus and sirolimus are potent and specific inhibitors of mTOR. At present, temsirolimus is approved by the European Medicines Agency (EMEA) and the Food and Drug Administration for treating advanced RCC. Of note, temsirolimus is a sirolimus ester that is hydrolyzed to sirolimus in patients, such that $74 \%$ of the circulating drug following weekly temsirolimus is actually sirolimus. At trough, circulating sirolimus concentrations exceed those of temsirolimus by approximately a factor of ten [23]. Intravenous administration of temsirolimus raises plasma concentrations of both temsirolimus and sirolimus which contribute antitumor activity after the administration of temsirolimus [24].

Screening for RCC is not recommended in the general population due to its rarity. Despite the incidence of RCC is increased in renal transplant recipients and, in particular, recipients with ACKD [8], the American Society of Transplantation has found no evidence to support regular screening using ultrasound or other radiology and urine cytology in renal transplant recipients [25]. On the other hand, Schwarz et al. [8] recommended a screening and management protocol in transplant recipients, incorporating the Bosniak renal cyst classification. Recently Goh et al. also advocated regular screening for renal cysts and RCC in ESRD patients both on transplant waiting list and after transplantation [12]. Inexpensive and noninvasive renal ultrasound still provides the best predictive value in screening and diagnosis of RCC in renal transplant recipients [26] with a positive predictive value of $100 \%$ $[3,27]$ and a negative predictive value of $92-94 \%$ [27, 28]. Goh et al. showed that routine ultrasound screening is already effective in making early diagnosis with excellent patient and graft outcomes in their center. They proposed that screening should be performed within a month of transplantation, then every 5 years of those without cysts and every 2 years of those with cysts so as to detect RCC early [12]. Although computerized tomography (CT) scan findings of RCC are similar for transplant recipients and general population, the presence of acquired multicystic renal dysplasia affects the diagnostic performance of CT scan. Terasawa et al. [29] and Sasagawa et al. [30] reported a RCC detection rate of only 67 and $71 \%$, respectively, with CT scan.
With the improvement of life expectancy, cancer now becomes a major cause of death following renal transplantation. The cost-effectiveness of cancer screening including RCC is still debatable. Currently, the decision to screen remains the choice of the managing physician without validated data and evidence to support such practices. With increasing data showing that a better prognosis of dialysis patients and general population with RCC if the malignancy is detected by screening rather than by the appearance of symptoms [31, 32], it is now time to revisit and reexamine the existing guidelines and to consider cost-effective screening policies in all kidney transplant candidates and recipients for native renal cysts and RCC. More detailed and uniform guidelines designed by the major international transplantation societies are probably necessary.

\section{Conflict of interest None.}

Open Access This article is distributed under the terms of the Creative Commons Attribution Noncommercial License which permits any noncommercial use, distribution, and reproduction in any medium, provided the original author(s) and source are credited.

\section{References}

1. Kasiske BL, Snyder JJ, Gilbertson DT, Wang C (2004) Cancer after kidney transplantation in the United States. Am J Transpl 4:905-913

2. Penn I (1999) Post-transplantation malignancies. Transpl Proc 31:1260-1262

3. Doublet J, Peraldi M, Gattegno B, Thibault P, Sraer JD (1997) Renal cell carcinoma of native kidneys: prospective study of 129 renal transplant patients. J Urol 158:42-44

4. American Joint Committee on Cancer (2002) Kidney. AJCC cancer staging manual, 6th edn. New York, Springer, pp 323-325

5. Kovacs G, Akhtar M, Beckwith BJ et al (1997) The Heidelberg classification of renal cell tumors. J Pathol 183:131-133

6. Fuhrman SA, Lasky LC, Limas C (1982) Prognostic significance of morphologic parameters in renal cell carcinoma. Am J Surg Pathol 6:655-663

7. Sheil A, Flavel S, Disney A, Mathew T (1985) Cancer development in patients progressing to dialysis and renal transplant. Transpl Proc 17:1685-1688

8. Schwarz A, Vatandaslar S, Merkel S, Haller H (2007) Renal cell carcinoma in transplant recipients with acquired cystic kidney disease. Clin J Am Soc Nephrol 2:750-756

9. Heinz-Peer G, Schoder M, Rand T, Mayer G, Mostbeck GH (1995) Prevalence of acquired cystic kidney disease and tumors in native kidneys of renal transplant recipients: a prospective US study. Radiology 195:667-671 
10. Hoshida Y, Nakanishi H, Shin M, Satoh T, Hanai J, Aozasa K (1999) Renal neoplasia in patients receiving dialysis and renal transplantation: clinico-pathological features and p53 gene mutation. Transplantation 68:385-390

11. Hughson MD, Buchwald D, Fox M (1986) Renal neoplasia and acquired cystic kidney disease in patients receiving long-term dialysis. Arch Pathol Lab Med 110:592-601

12. Goh A, Vathsala A (2011) Native renal cysts and dialysis duration are risk factors for renal cell carcinoma in renal transplant recipients. Am J Transplant 11:86-92

13. Filocamo MT, Zanazzi M, Li Marzi V et al (2009) Renal cell carcinoma of native kidney after renal transplantation: clinical relevance of early detection. Transpl Proc 41: 4197-4201

14. Neuzillet Y, Lay F, Luccioni A et al (2005) De novo renal cell carcinoma of native kidney in renal transplant recipients. Cancer 103:251-257

15. Luciani LG, Cestari R, Tallarigo C (2000) Incidental renal cell carcinoma-age and stage characterization and clinical implications: study of 1092 patients (1982-1997). Urology 56:58-62

16. Penn I (1995) Primary kidney tumors before and after renal transplantation. Transplantation 59:480-485

17. Hoshida Y, Tsukuma H, Yasunaga Y et al (1997) Cancer risk after renal transplantation in Japan. Int $\mathbf{J}$ Cancer 71:517-520

18. Ishikawa N, Tanabe K, Tokumoto T et al (1998) Renal cell carcinoma of native kidneys in renal transplant recipients. Transpl Proc 30:3156-3158

19. Hughson MD, Bigler S, Dickman K, Kovacs G (1999) Renal cell carcinoma of end stage renal disease: an analysis of chromosome 3, 7, and 17 abnormalities by microsatellite amplification. Mod Pathol 12:301-309

20. Campbell SC, Novick AC, Belldegrun A et al (2009) Guideline for management of the clinical T1 renal mass. J Urol 182:1271-1279

21. Guba M, von Breitenbuch P, Steinbauer M et al (2002) Rapamycin inhibits primary and metastatic tumor growth by antiangiogenesis: involvement of vascular endothelial growth factor. Nat Med 8:128-135
22. Campistol JM (2009) Minimizing the risk of posttransplant malignancy. Transplantation 87:S19-S22

23. Brugarolas J, Lotan Y, Watumull L, Kabbani W (2008) Sirolimus in metastatic renal cell carcinoma. J Clin Oncol 26:3457-3460

24. Hudes G, Carducci M, Tomczak P et al (2007) Global ARCC Trial. Temsirolimus, interferon alpha, or both for advanced renal-cell carcinoma. $N$ Engl J Med 356: 2271-2281

25. Kasiske BL, Vazquez MA, Harmon WE et al. (2000) The American society of transplantation: recommendations for the outpatient surveillance of renal transplant recipients. J Am Soc Nephrol 11(Suppl 15): S1-86

26. Wiesel M, Carl S, Drehmer I, Hofmann WJ, Zeier M, Staehler G (1997) The clinical significance of renal cell carcinoma in dialysis dependent patients in comparison with kidney transplant recipients. Urologe A 36:126-129

27. Moudouni SM, Lakmichi A, Tligui M et al (2006) Renal cell carcinoma of native kidney in renal transplant recipients. BJU Int 98:298-302

28. Schmidt R, Stippel D, Krings F, Pollok M (1995) Malignancies of the genito-urinary system following renal transplantation. Br J Urol 75:572-577

29. Terasawa Y, Suzuki Y, Morita M, Kato M, Suzuki K, Sekino H (1994) Ultrasonic diagnosis of renal cell carcinoma in hemodialysis patients. J Urol 152:846-851

30. Sasagawa I, Terazawa Y, Ishizagi M et al (1992) Comparison of ultrasonography, computerized tomography and angiography in dialysis patients with renal cell carcinoma. Urol Int 49:206-210

31. Thompson IM, Peck M (1988) Improvement in survival of patients with renal cell carcinoma: the role of the serendipitously detected tumor. J Urol 140:487-490

32. Ishikawa I, Honda R, Yamada Y, Kakuma T (2004) Renal cell carcinoma detected by screening shows better patient survival than that detected following symptoms in dialysis patients. Ther Apher Dial 8:468-473 